\title{
Are there any significant craniomaxillofacial manifestations of COVID-19?
}

\section{Vaibhav Sahni'}

\section{A commentary on}

\section{Pitak-Arnnop $\mathbf{P}$, Meningaud J-P, Sirintawat $\mathbf{N}$ et al.}

A German AWMF's S2e/realist synthesis and meta-narrative snapshot of craniomaxillofacial manifestations in COVID-19 patients: Rapid living update on 1 January 2021. I Stomatol Oral Maxillofac Surg 2021; DOI: 10.1016/j.jormas.2021.01.012.

\begin{abstract}
Data sources PubMed/Medline, Biomed Central, Embase, Thai Journals and the Cochrane library.

Study selection Reports or studies dealing with the craniomaxillofacial (CMF) manifestations of COVID-19 were selected. Data extraction and synthesis The reviewers extracted data from the included studies; however, being a meta-narrative review, it was not deemed fit to contact authors for unpublished work, conduct a meta-analysis, search for unpublished data or search for studies in languages different from English, German, French and Thai. The primary variable for prediction was set as the COVID-19 infection, with the primary outcome variable being manifestation in the CMF region. Parameters of a clinical, demographic, investigative and therapeutic nature were the other included variables.

Subsequently, the reports and abstracted data were reviewed for study design, author names, country, size of the sample, level of evidence, signs/symptoms, treatment undertaken and investigations conducted. Studies exhibiting the highest level of evidence were included eventually. As deemed appropriate, uni/bivariate and descriptive statistics were applied.

Results Cumulatively, 101 publications were included in the metanarrative after due screening, the data of which was further divided into five groups of: 1) nose/paranasal sinus/skull base; 2 ) mouth/ throat; 3) periorbital/ocular tissue; 4) skin; and 5) ear.

Conclusions The majority of data pertaining to CMF manifestations of COVID-19 qualifies as a low level of evidence. With the exception of taste/smell dysfunction, most CMF manifestations are nonspecific and can be diagnosed by CMF surgeons and dentists, which underscores their role in battling the pandemic.
\end{abstract}

\section{Commentary}

The review found that craniomaxillofacial (CMF) signs and symptoms were a definite occurrence in relation to COVID-19 infection. The evidence for this claim was, however, of low quality. The authors found that the majority of reported data involved the assessment of taste and smell disturbances in patients afflicted with COVID-19. Apart from these, other CMF signs and symptoms tended to be rather nonspecific in nature and presentation.

\section{GRADE rating}

\section{Practice points}

- Dental practitioners should be aware of the various signs and symptoms related to COVID-19 in the CMF region along with their management.

- Knowledge of CMF manifestations of COVID-19 would enhance timely referral and make dentists and CMF surgeons active players in COVID-19 management.

The management of these lesions involved the institution of conservative measures, which generally sufficed as a form of patient care.

The authors mentioned various mechanisms by which different CMF regions seemed to become involved in the COVID-19 infection process to subsequently present as various signs and symptoms.

\section{Mouth and throat}

SARS-CoV-2 is implicated in specifically targeting angiotensin converting enzyme 2 (ACE2) receptors. These have been found to be present in the mucosa of the tongue, salivary gland tissue and gingiva. This interaction purportedly produces cytokine release syndrome. The mechanism of entry into the target cells involved ACE2 and TMPRSS2, its serine protease. This viral-ACE2 receptor interaction causes an impairment of the sensitivity of taste buds and an overall disturbance in gustatory response. Viral-mediated impairment of the peripheral nervous system, viral binding to mucin components of the saliva (thereby causing accelerated taste particle degradation) and gustatory function form the other proposed components of the SARS-CoV-2 pathophysiology.

\section{Nose/paranasal sinus and skull base}

An increased expression of ACE2 receptors in relation to the nasal mucosa tends towards higher viral loads in the nasal cavity. Nasal pathologies have also been observed to be a hindrance to nasal sampling and lead to false-negative results. Rhinosinusitis has not been observed to differ in its prevalence among patients suffering from COVID-19 and those without this affliction. At the same time, however, it has been noted that chronic rhinosinusitis and pre-existing atopy predisposed patients to be spreaders for longer periods of time and required protracted periods of quarantine. Disturbance of smell as a heralding symptom has been related to an increase in IL 6 levels. SARS-CoV-2 interaction with olfactory ACE2 receptors may also prevent the passage of odoriferous molecules to the olfactory epithelium. Intracranial viral spread has been proposed to occur via the transcribral route, the trigeminal route or the phrenic nerve route.

\section{Periorbital/ocular tissue}

TMPRSS2-mediated ACE2 interaction with SARS-CoV-2 has been implicated in viral spread from the nasolacrimal duct and sinonasal 
region. Despite early data suggesting ocular complications as being common, the authors have cited the prevalence to be quite low. Xerophthalmia tends to increase irrespective of ventilation and intensive care. Conjunctivitis tended to increase in patients with taste and smell disturbances. Patients with severe pneumonia were noted to have increased prevalence of orbital/ocular COVID-19related manifestations. Haematogenous or neural spread has been implicated in the development of acute neuro-ophthalmic diseases. Ocular/orbital changes as a result of SARS-CoV-2 can confound assessment in patients after midface/orbital fracture treatment. Iatrogenic lesions, such as those due to medications and respirator usage, have also been observed. Household violence as a result of prolonged lockdowns also tended to increase the presence of ocular/ orbital trauma.

Ear

Sudden sensorineural hearing loss may occur as a result of direct labyrinthine or cochlear nerve invasion, latent virus reactivation or as a result of host immune response.

\section{Skin}

C4d, C5b-9 and MASP2 complex mediators may induce damage to the cells of the epithelium and subsequently cause an activation of clotting factors to culminate into disseminated intravascular coagulation. Only singular reports of necrotising periodontal infection and lip necrosis were reported in the literature, leading the authors to remark that oral and facial tissues may indeed be spared COVID-19-related arterial occlusive disease.

\section{Concluding remarks}

The authors acknowledged that the addition of several databases and publications in languages other than English did not offset the obvious flaw of the literature base being of low quality.

Apart from signs and symptoms which resulted as a direct manifestation of COVID-19 infection, the authors also pointed out a number of conditions which could arise as a possible fallout of patient care practices. These included acute macroglossia arising from prolonged prone positioning of patients and orbital compartment syndrome as a result of protracted ventilator dependence.

The authors acknowledged that close collaboration among specialities dealing with the examination of the mouth and the CMF region as a whole would go a long way in mitigating the impact of the pandemic.

In short, the study identified a number of CMF signs and symptoms related to COVID-19. Except taste and smell disturbances, these were nonspecific in nature and were managed conservatively. The evidence for these findings was, however, low.

\section{Author affiliation}

${ }^{1}$ Panjab University, Chandigarh, Vasant Kunj, New Delhi, India. Correspondence to: Vaibhav Sahni

Evidence-Based Dentistry (2021) 22, 72-73.

https://doi.org/ 10.1038/s41432-021-0177-z 